\title{
Managing eosinophilic esophagitis: challenges and solutions
}

\author{
Nisha A Shah \\ Dustin MAlbert \\ Noah M Hall \\ Fouad J Moawad
}

Department of Medicine, Gastroenterology Service, Walter Reed National Military Medical Center, Bethesda, MD, USA

Correspondence: Fouad J Moawad

Gastroenterology Service, Department of Medicine, Walter Reed National Military Medical Center, 890I Wisconsin Avenue, Bethesda, MD 20889, USA

$\mathrm{Tel}+\mathrm{I} 30 \mathrm{I} 2954600$

Fax + I 30I 2954599

Email moawad.fouad@scrippshealth.org
This article was published in the following Dove Press journal:

Clinical and Experimental Gastroenterology

16 September 2016

Number of times this article has been viewed

\begin{abstract}
Eosinophilic esophagitis (EoE) is a chronic and progressive immune-mediated condition defined by symptoms of esophageal dysfunction and dense eosinophilic infiltration of the esophageal mucosa. Therapies consist of anti-eosinophilic medications and specialized diets aimed to decrease the progression of EoE and alleviate its symptoms, namely, dysphagia and food impaction. Assessing response to therapy remains challenging, as treatment end points are not well defined and currently consist of clinical, histologic, and endoscopic features. Newer validated measures may help standardize treatment end points. Emerging data support the use of maintenance therapy, which may reduce disease progression. Optimal dosages, delivery techniques, and duration of treatment need to be determined. When features of fibrostenosis develop, esophageal dilation is a safe and effective adjunctive strategy for improving symptoms. In EoE cases refractory to conventional treatments, newer therapies targeting inflammatory mediators and cytokines are on the horizon.
\end{abstract}

Keywords: eosinophilic esophagitis, esophagitis, eosinophilia, dysphagia, allergy, corticosteroids, dietary treatment, dilation

\section{Introduction}

Eosinophilic esophagitis (EoE) is defined by symptoms of esophageal dysfunction, namely, dysphagia and food impaction in adults, in the presence of dense eosinophilic infiltration of the esophageal mucosa. ${ }^{1}$ EoE is a chronic progressive immune-mediated condition, without evidence of neoplastic transformation, ${ }^{2,3}$ that affects the quality of life and may lead to frequent emergency room visits due to food impactions. ${ }^{4,5}$ During the past 2 decades, emerging literature has improved the understanding of the pathogenesis of EoE, in turn leading to better treatment options.

There are three main strategies in the management of EoE: drugs, specialized diets, and dilation when esophageal strictures develop (Figure 1). ${ }^{1,6,7}$ Despite the different treatment options, there is no clear consensus to define end points of therapy. The current method of assessing therapeutic success is burdensome as it involves repetitive endoscopic evaluations with biopsies. One of the biggest challenges remains the management of refractory EoE, yet newer therapies are on the horizon.

\section{Treatment end points}

From a patient's perspective, the most important treatment end point is improvement of clinical symptoms and quality of life. From a clinician's standpoint, reducing histologic and endoscopic inflammation with medications or specialized diets is a main 


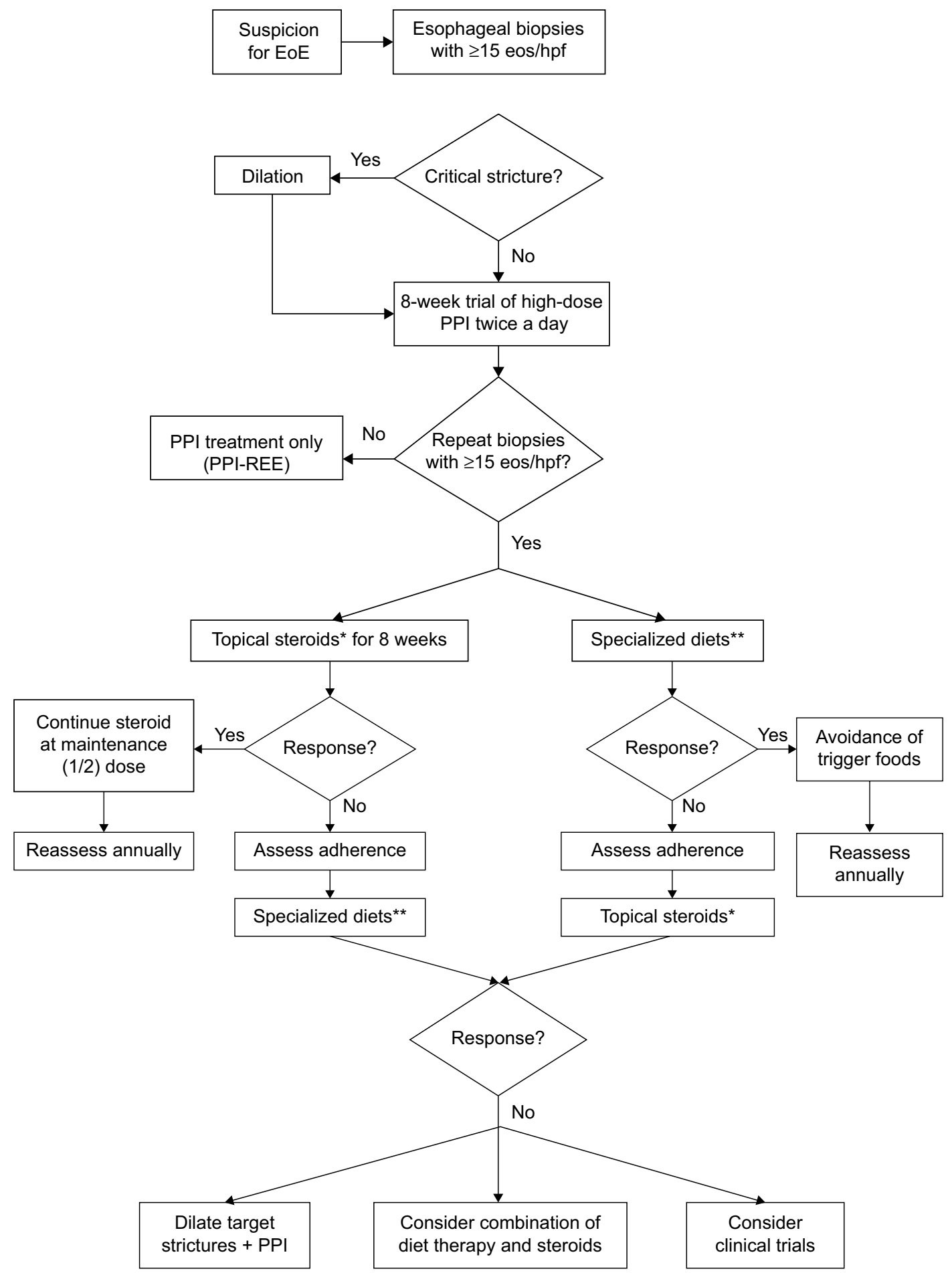

Figure I Treatment algorithm for EoE.

Notes: *Swallowed fluticasone or budesonide; **six-food elimination diet, four-food group elimination diet, elemental diet, allergy-test targeted elimination diet. Abbreviations: EoE, eosinophilic esophagitis; eos, eosinophils; hpf, high-power field; PPI, proton pump inhibitor; REE, responsive esophageal eosinophilia.

treatment goal, which should translate to improved symptoms. Unfortunately, only modest concordance exists between symptoms and histology. ${ }^{8,9}$ Several treatment outcomes now have validated instruments and are available for use in both the clinical and research settings. ${ }^{4,10,11}$

The most commonly used outcome to assess treatment response in EoE is measurement of eosinophil count.
However, other histologic variables such as microabscesses, basal cell hyperplasia, spongiosis, and progressive lamina propria fibrosis do exist. ${ }^{12}$ Even though measuring eosinophil counts before and after treatment seems objective, challenges exist in standardizing histology as a treatment end point. First, a large discrepancy exists in the literature with regard to using quantitative cutoffs. Studies have defined histologic 
response as achieving an absolute eosinophil count (from $\leq 1$ to $<15$ eosinophils [eos]/high-power field [hpf]) to a reduction in the percentage of pretreatment eosinophils. ${ }^{13-17}$ Second, variability exists in the area under the microscope and the methods followed for counting of eosinophils. ${ }^{18}$ Some studies use peak eosinophilia while other studies use a mean count of several fields. ${ }^{18}$ Third, EoE manifests as a patchy condition and some biopsies may show more inflammation than others. ${ }^{19}$ Another limitation of using histology as an end point in EoE is its poor predictably of clinical response. ${ }^{17,20-22}$ Recently, a validated histology score was published which acknowledges the limitations of relying solely on eosinophil peak count. ${ }^{10}$ This score takes into account eight histologic factors that are scored based on severity and extent of inflammation. One particular benefit of this scoring system is that composite histology scores strongly correlate with treatment status.

Another commonly used treatment end point is change in clinical symptoms. Several questionnaires have been used in interventional studies, but only two instruments have been validated for EoE: the Eosinophilic Esophagitis Activity Index (EEsAI) and the Dysphagia Symptom Questionnaire (DSQ). ${ }^{23,24}$ The DSQ is a simple three-question survey based on patient-reported outcomes. ${ }^{24}$ EEsAI was developed by a group of international experts in EoE. It assesses dysphagia to eight different food textures in addition to behavior modification over a 7-day recall period. Although the EEsAI correlated well with endoscopic features in patients with severe symptoms, it was less sensitive in patients with mild to moderate symptoms. ${ }^{9}$ The Mayo Dysphagia Questionnaire has been used in studies but has not been validated for EoE. ${ }^{25,26}$ The Straumann Dysphagia Index, which assesses frequency and intensity of dysphagia, was used in one randomized controlled trial comparing budesonide with placebo. ${ }^{27}$ Clinical improvement alone, though, does not predict regression of disease. One potential reason why only modest concordance exists between the degree of eosinophilia and clinical symptoms may be due to the involvement of other inflammatory cells besides eosinophils. A recent study described a small cohort of patients with EoE-like disease, who presented identically to EoE but without histological evidence of eosinophils in the esophagus. ${ }^{28}$

Changes in endoscopic features have been used as end points to therapy as well. A recent prospective study evaluated the accuracy of EoE Endoscopic Reference Score (EREFS), a scoring system to diagnose and monitor therapy. ${ }^{11}$ EREFS correlated well with treatment response to steroids $(P<0.001)$, and responders to treatment had a significant reduction in score compared to nonresponders. ${ }^{9}$ The predictive ability of
EREFS score was better for inflammatory (furrows, exudates, edema) compared to fibrostenotic features. ${ }^{9}$ Another study used EREFS before and after treatment with either medications or diet and found a significant reduction in score among responders to therapy. ${ }^{29}$

Although limited to specialized centers and research settings, assessing esophageal epithelial barrier integrity is a promising treatment end point. It plays a role in antigen exposure, thus leading to the inflammatory cascade. Expression of desmoglein-1 and filaggrin marker genes correlated with improvement of barrier integrity. ${ }^{30}$

Despite the availability of multiple end points, each treatment end point individually has limitations. For this reason and since EoE involves a clinical-histological diagnosis with distinct endoscopic features, therapeutic end points should continue to reflect a combination of histology, clinical symptoms, and endoscopic remission.

\section{Role of acid suppression in EoE}

The efficacy of proton pump inhibitors (PPIs) for the treatment of esophageal eosinophilia has been well established in multiple studies. ${ }^{26,31,32}$ A recent systematic review of 33 studies found that over $60 \%$ of patients treated with PPI had a clinical response and 50\% achieved histologic remission. ${ }^{33}$ While there has long been debate about whether PPIresponsive esophageal eosinophilia (PPI-REE) should be considered a separate entity from EoE, a recent international task force proposed that PPI-REE should be viewed as part of the EoE spectrum and that PPIs should be considered as first-line treatment. ${ }^{34}$

Studies have demonstrated that PPI-REE and EoE are virtually identical clinically, endoscopically, histologically, and even on a molecular level. ${ }^{35,36,37}$ One study found similar levels of tryptase and eotaxin-3 tissue biomarkers in EoE and PPI-REE, both of which were significantly different from controls. ${ }^{38}$ Similarly, one study found no significant difference in eotaxin-3 staining between EoE and PPI-REE, but a significant difference was observed when compared to gastroesophageal reflux disease patients. ${ }^{36}$ Another study analyzed transcriptome heat maps which showed significant overlap between PPI-REE and EoE. ${ }^{37}$ Both expressed genes involved in chemotaxis, mast cell function, and tissue remodeling and protection. PPI therapy demonstrated reversal of expression in the PPI-REE group. ${ }^{37}$ Interestingly, a potassium ion channel (KCNJ2) was found to be the only gene exhibiting a significant difference in expression in PPI-REE compared to EoE patients. ${ }^{37}$ However, the factors that dictate success with PPI remain to be elucidated, and at present, only response itself to treatment can distinguish between the phenotypes. ${ }^{39}$ 
Despite this consensus proposal, the dosage, frequency, duration of therapy, and optimal interval to assess response remain unclear. A recent review of nine clinical trials published between 2010 and 2015 reported response rates to PPI ranging from $36 \%$ to $69 \%$, with histologic response defined by achieving $<15$ eos/hpf; however, slightly lower rates $(25 \%-49 \%)$ were noted when stricter criteria were employed. The most commonly studied regimen, accounting for six of the nine trials, included the administration of omeprazole or esomeprazole $40 \mathrm{mg}$ twice daily for 8 weeks. Similar response rates were observed in studies using $20 \mathrm{mg}$ twice-daily dosing is given for a period of 6 weeks. ${ }^{34}$

Recent studies have raised insight into the mechanism of how acid suppressants may work in an allergic-mediated condition. PPIs have both antisecretory and anti-inflammatory effects. ${ }^{40}$ Acid reflux is a trigger for eosinophilia and suppression of acid improves inflammation. In one study, using tissue impedance levels, PPI therapy was effective in partially restoring the integrity of the epithelial barrier to reflux. ${ }^{41}$ There is also limited evidence demonstrating that PPIs have a direct effect on preventing eosinophil migration into the esophageal mucosa ${ }^{42}$ This is secondary to inhibition of eotaxin-3 induced by $\mathrm{TH}_{2}$, interleukin-4 (IL-4), and IL-13. ${ }^{43}$ It remains unclear, however, why some patients respond to treatment while others do not.

Although some patients lose response to PPI over time, the majority continue to maintain improvement in symptoms and histology. ${ }^{44}$ One recent study demonstrated that over $70 \%$ of patients with esophageal eosinophilia showed a sustained response when followed for over 2 years. Interestingly, many of those who lost response to treatment were found to be positive for CYP2C19, a gene associated with rapid metabolism of PPIs. Moreover, $90 \%$ of these patients regained response with simple dose escalation of PPI. ${ }^{45}$

\section{Phenotypes}

There are two phenotypes that are described for EoE. ${ }^{4,29,46,47} \mathrm{In}$ its earlier stages, EoE is more commonly recognized as an inflammatory condition, characterized by the endoscopic appearance of linear furrows, edema, white plaques, and without visible esophageal remodeling. This phenotype is more prevalent in children, which suggests that the duration of disease is an important contributor to esophageal remodeling. After several years, and possibly due to lack of treatment, features of fibrostenosis develop. This is defined by the presence of trachealization, a diffusely narrow esophagus, and dominant stricture formation. ${ }^{4,48}$ These two phenotypes can coexist, and in one study, a mixed phenotype was noted in the majority of EoE patients over time. ${ }^{4}$

One recent study reported that some EoE patients have an extremely narrow esophagus, defined as requiring a neonatal endoscope to traverse the stricture. The patients of this subphenotype had a longer duration of disease and were less responsive to topical steroids compared to those without extremely narrow strictures $(P<0.01)$. In these patients, dilation should be used early in treatment in conjunction with medical or dietary therapy. ${ }^{49}$

A newly described condition, referred to as "EoE-like" disease, appears to be another phenotype of EoE in which patients present identically to EoE, but without eosinophils on biopsies. This phenotype is not only a diagnostic challenge but also opens new insights into the pathogenesis of EoE. ${ }^{28}$

\section{Topical corticosteroids}

Topical steroids are first-line therapy for EoE and can induce remission of esophageal inflammation through inhibition of proinflammatory cytokines. ${ }^{1,50}$ Swallowed aerosolized fluticasone and viscous budesonide are the two formulations currently used in practice.

In open-label trials, fluticasone demonstrated favorable histologic responses in over $90 \%$ of the cases. ${ }^{51,52,53}$ Despite initial promising reports, randomized controlled trials in both children and adults did not yield similar therapeutic success. In a randomized controlled trial in pediatric EoE patients, fluticasone induced remission in $50 \%$ of patients..$^{13}$ In two randomized controlled trials of PPI-naïve adult EoE patients, treatment with fluticasone $440 \mu \mathrm{g}$ twice daily for 8 weeks in patients demonstrated a histologic response of $15 \%$ and $19 \%$, defined as $\leq 5 \mathrm{eos} / \mathrm{hpf}$ and $\leq 7 \mathrm{eos} / \mathrm{hpf}$, respectively. ${ }^{14,26} \mathrm{In}$ EoE patients unresponsive to PPI therapy, higher-dose fluticasone (1,760 $\mu \mathrm{g}$ daily) for 6 weeks led to histologic response in $62 \%$ of adult EoE patients, defined as a greater than $90 \%$ decrease in pretreatment eosinophilia. ${ }^{17}$ Similarly, in a mixed population of children and adults, higher-dose fluticasone resulted in histologic response in $65 \%$ of patients. ${ }^{54}$ These two studies suggested that response to fluticasone is dose dependent, as was noted in two recent studies where dose escalation induced a better response. ${ }^{15,55}$ Interestingly, the study by Butz et al also suggested that nonresponders to treatment had evidence of steroid resistance based on molecular gene expression. ${ }^{54}$

Budesonide can be administered through swallowing a nebulized form, as an effervescent tablet, or by swallowing a viscous slurry created with a sugar substitute. Histologic and clinical remission was achieved in 18 patients in a randomized, double-blind, placebo-controlled trial using a 
nebulized suspension of $1 \mathrm{mg}$ budesonide twice daily for 15 days. ${ }^{27}$ Due to improved mucosal contact time, viscous solution showed better histologic response compared to nebulized form in a single study and is more commonly used in clinical practice. ${ }^{56}$ Controlled studies exist in children and adults, but differ in duration of treatment and definitions of histologic response. The response rates in these trials are higher compared to those carried out with fluticasone ( $52 \%-86 \%)$, although there are no prospective studies directly comparing the two formulations. ${ }^{57,58}$ A retrospective review of $75 \mathrm{EoE}$ patients compared the efficacy of fluticasone $440 \mu \mathrm{g}$ twice daily to budesonide $1 \mathrm{mg}$ twice daily for 8 weeks. There was no significant difference in histologic response, defined as $<15$ eos/hpf ( $48 \%$ vs $56 \%$, $P=0.632) .{ }^{59}$ Although some correlation exists between clinical symptoms and histologic response, the degree of eosinophilia needed to induce a symptomatic response is unknown.

There is a difference in the preparation and administration of both steroids. Patients are instructed to puff and swallow an aerosolized formulation of fluticasone. This may cause decreased mucosal contact time and absorption is likely not uniform among patients. Budesonide is prepared in the form of a viscous slurry by mixing aqueous respules with either honey or xanthan gum. Further, eating or drinking should be avoided for 30-60 minutes after administration to reduce the possibility of early drug clearance. It is important to note that current pharmacologic therapies come with specific instructions that if not followed could negatively impact therapy.

Topical steroids are safe with few reported side effects. They are most commonly reported side effect is candida esophagitis, occurring in up to $30 \%$ of cases, which appears to be dose dependent. ${ }^{1,17}$ Most cases are asymptomatic and discovered incidentally on repeat endoscopy. In one study, no adrenal suppression was reported, while in another suboptimal cortisol stimulation was reported in a few cases. ${ }^{60,61}$

Response to corticosteroids in EoE is lower than would be expected for an allergic immune-mediated condition. Knowledge of predictors of response may help guide patient counseling in choosing therapy by increasing steroid dosage or by focusing on alternative treatment options. Children with EoE who had more inflammation at baseline were shown to exhibit a greater response to fluticasone. ${ }^{62}$ Baseline dilation was found to be a predictor of response to steroids in a single study but was not reproduced in another study with a smaller sample size. ${ }^{59,63}$ Furthermore, clinical presentation or endoscopic features were not predictors of nonresponse. ${ }^{59}$

\section{Maintenance vs on-demand treatment with topical steroids}

In the majority of EoE patients, symptoms and esophageal inflammation recur following cessation of either medical or dietary therapy. Ongoing histologic inflammation can lead to esophageal remodeling with ensuing fibrosis, angiogenesis, mural thickening, and decreased esophageal wall elasticity. ${ }^{64,65}$ From a phenotypic perspective, data suggest that untreated EoE can progress from inflammatory to fibrostenotic disease with stricture formation and food impactions. ${ }^{4}$, ${ }^{46}$ To halt progression or at least delay it, maintenance therapy is recommended; however, there is limited data to support this hypothesis.

In a randomized double-blind placebo-controlled study, 28 patients were treated with $0.5 \mathrm{mg}$ of a nebulized budesonide suspension daily for 50 weeks after initially achieving histologic and symptomatic remission with budesonide $2 \mathrm{mg}$ daily. Subjects were instructed to nebulize the suspension of budesonide into the oral cavity and swallow continuously for $\sim 5$ minutes. Approximately two-thirds maintained clinical remission and 50\% maintained histologic remission, and the study was able to demonstrate a trend toward normalization of initial esophageal remodeling. ${ }^{66}$ Budesonide may also decrease esophageal remodeling after 3 months of therapy. ${ }^{67}$ In another study addressing short-term maintenance therapy, $93 \%$ of patients continued to maintain response, defined as $<15$ eos/hpf, with half the dose of swallowed fluticasone. ${ }^{54}$ A retrospective multivariate analysis of adults with 5 years of follow-up demonstrated a significant association between duration of treatment with topical steroids and reduced risk for food impactions. ${ }^{5}$ These data are encouraging for maintenance therapy; however, questions remain regarding duration of therapy, optimal dosing, and frequency of follow-up.

\section{Specialized diets}

A dietary trigger can induce an antigen or immune-mediated response in EoE. Specialized diets offer a nonpharmacologic option that targets the underlying disease process. The three dietary management options include elemental diet, targeted elimination diet based on skin prick and patch testing, and empiric food elimination diets.

Aminoacid-based elemental diets are very effective in inducing a histologic response in EoE patients, as their use eliminates the most common dietary triggers. ${ }^{68,69} \mathrm{~A}$ metaanalysis of 13 pediatric studies demonstrated up to $90 \%$ histologic remission in EoE patients. ${ }^{68}$ Data for use in adults with EoE, however, are more scarce. A single adult randomized controlled study included 29 subjects of whom $38 \%$ did 
not adhere to the diet. ${ }^{70}$ Of the 18 compliant patients, $72 \%$ achieved histologic response. EoE recurred in all patients 1 week after food was reintroduced. ${ }^{70}$ Two of the aminoacidbased diets used in these studies were Neocate 1 (SHS North America, Gaithersburg, MD, USA), consisting of free amino acids, corn syrup solids, and medium-chain triglyceride oil, and EleCare Free Amino Acid-based Medical Food (Abbott Nutrition, Abbott Laboratories, Columbus, OH, USA), another clinically proven hypoallergenic formula.

In children, elemental diets may require administration through a feeding tube. This diet is expensive, has poor palatability, and can be socially limiting, especially in adults. ${ }^{71}$ This diet, though, is effective and should be considered in those who have more severe disease or have failed other therapies.

A second dietary option is the test-directed elimination diet. Allergy testing using skin prick and patch tests identifies potential triggers that can be avoided with an overall efficacy of $\sim 48 \%{ }^{68}$ Data in adults indicate that this method is less robust and less efficacious in inducing histologic remission, reported to be $\sim 32 \%$, with better clinical improvement. ${ }^{68}$ Similar to elemental diets, most data are in the pediatric population. ${ }^{72}$ In children, there is a wide variability in response rates ranging from $37 \%$ to $77 \%$ compared to histologic remission based on one of the larger adult studies. ${ }^{68,73,74}$

The role of allergy testing is less clear due to variations in positive and negative predictive values. In one study including adults, $67 \%$ of subjects who had a food trigger identified by reintroduction had a negative skin prick test to all foods, while skin prick testing predicted only $13 \%$ of food triggers. ${ }^{16}$ In a single study of 941 pediatric patients, the combination of skin prick tests and atopy patch tests had a negative predictive value of $92 \%$, except for milk which was $44 \%$. However, the positive predictive value in this study was low at $44 \% .^{75}$ This differs from another study of 98 pediatric patients in which the negative predictive value of skin testing ranged from $40 \%$ to $67 \%$ depending on the source (milk, $40 \%$; egg, $56 \%$; soy, $64 \%$; wheat, $67 \%) .{ }^{76}$ Knowledge of positive and negative predictive values should be taken into consideration when interpreting results of allergy testing and while counseling patients. The validity and standardization of this approach should be further studied before implementing in clinical practice.

The six-food elimination diet (SFED) potentially overcomes some of the limitations associated with the previous two specialized diets. Patients eliminate the six most commonly encountered food allergens: milk, wheat, soy, nuts, egg, and seafood. Compared to the previous two dietary approaches, this diet has been well studied in adults with homogeneity of results across well-designed studies. ${ }^{16,77}$ The overall efficacy was $73 \%$ in children and $71 \%$ in adults. ${ }^{68}$ In a study of 67 adults, $73 \%$ achieved histologic response, defined as $<15$ eos/hpf. ${ }^{77}$ Twenty-five patients were available for follow-up at 1 year and all maintained histologic remission and remained asymptomatic. Fifteen patients were followed up at 2 years and four patients at 3 years. All remained in histologic remission and were asymptomatic without need for pharmacologic treatment. ${ }^{77}$ Although the unpredictability of allergy testing is avoided, this approach can be costly and labor intensive when searching for the dietary trigger. If the protocol is strictly followed, an esophagogastroduodenoscopy is performed every 6 weeks after food is reintroduced to assess for histological response.

Approximately $85 \%$ of patients will have one or two food triggers identified after food reintroduction with the SFED. To limit this, a four-food group elimination diet (FFGED, dairy products, wheat, egg, and legumes) was studied in 52 adults who were assessed for clinical and histologic remission after 6 weeks. Clinicopathologic remission was achieved in 54\%, and $31 \%(6 / 19)$ of nonresponders were successfully rescued with the SFED. Only one or two food triggers were identified in all patients, with milk being the sole trigger in $27 \% .{ }^{78} \mathrm{In}$ most cases, strict removal of multiple foods is temporary. The primary and long-term goal is to identify and eliminate one or a few dietary triggers allowing patients to introduce nonallergenic sources of food. This dietary approach requires a motivated patient and can initially have a negative impact on quality of life, but clinical improvement should offset this first hurdle. When successful, it allows the possibility of long-term maintenance with avoidance of medications and provides patients with control over their condition.

\section{Dilation}

Some years ago, a letter to the editor concluded that dilation "should not be considered in patients with eosinophilic esophagitis", ${ }^{79}$ a prevailing thought 1 decade ago owing to mucosal fragility of EoE, dire appearance of post-dilation deep mucosal tears, and numerous case reports of esophageal perforation. ${ }^{80}$ Since dilation does not alter the underlying pathogeneses of EoE, the most recent guidelines recommend against dilation as first-choice treatment except in certain cases. ${ }^{1}$ However, data exist in small studies in which EoE patients can maintain clinical remission over several years with only PPI and dilation. ${ }^{2,64}$

Two studies demonstrate longevity of dilation for clinical symptoms in steroid-naïve patients. The first study followed 
30 patients for a mean of 7.2 years in which eleven EoE patients required dilations. In these patients, $60 \%$ were symptom free for an average of 8.6 months. ${ }^{64}$ The second study was a retrospective single institution study of $13 \mathrm{EoE}$ patients followed for a mean of 13 years in which dilation was performed every 2 years to maintain a lumen diameter of $17 \mathrm{~mm} .^{2}$ One retrospective study found $67 \%$ of patients had symptoms requiring repeat dilation every 15 months. ${ }^{81}$ A systematic review reported a period of clinical response lasting between 3 and 23 months. ${ }^{82}$ In one meta-analysis, $75 \%$ of EoE patients showed clinical improvement following dilation. ${ }^{83}$

Expanding esophageal diameter is an important treatment goal of dilation in EoE. When esophageal diameter increased from 12 to $16 \mathrm{~mm}, 83 \%$ showed significant clinical improvement, $P<0.001 .^{84}$ In a study with the longest followup to date, $87 \%$ of patients showed symptom response after a mean esophageal lumen diameter of $15.2 \mathrm{~mm}$ was reached; however, 58\% required repeat dilations within 12 months, often with the second dilation 4 months after the initial procedure. ${ }^{64}$ In surveys, $90 \%$ of patients reported mild to no dysphagia when esophageal diameter was extended to 16-17 $\mathrm{mm}$. This resulted in improvement of dysphagia in over $80 \%$ of patients for up to 6 months and $67 \%$ by 12 months. ${ }^{81}$ Most studies support the use of dilation for symptomatic response with the understanding that repeat dilations are likely required within $1-2$ years. ${ }^{2,83}$

Several studies have assessed the safety of esophageal dilation in EoE. In a large cohort of 207 dilations, there were no perforations or episodes of major bleeding following dilation.$^{81}$ However, $74 \%$ of patients complained of post-procedural chest pain in questionnaires, $80 \%$ reported the pain as minimal to none, and all said they would repeat the procedure. Another large cohort from a single center examined 161 patients undergoing 293 dilations and reported three perforations. The first two perforations occurred with a 45-French Savary dilator with an estimated esophageal lumen diameter of $9 \mathrm{~mm} \cdot{ }^{85} \mathrm{In}$ this study, the frequency of major bleeding was $0.3 \%$, and deep mucosal tears were $9.2 \%$. The authors reported multiple dilations (odds ratio [OR] $3.7,95 \%$ confidence interval [CI] 1.73-7.91) and location of narrowing (OR 5.62, 95\% CI 2.07-15.26) as risk factors for developing complications. In a study by Dellon et al, the five reported complications of deep mucosa tears and chest pain were also associated with multiple dilations $(P=0.009) .{ }^{84}$ However, it is debatable whether deep mucosal rents and chest pain constitute actual complications, as a tear is an intended outcome following dilation, and several patients develop self-limited chest pain following the procedure. In a recent retrospective study of $164 \mathrm{EoE}$ patients who underwent a total of 486 dilations, the overall complication rate was $5 \% .{ }^{86}$ There were no reported cases of perforations or hemorrhage that were noted; however, $4.3 \%$ experienced post-procedure chest pain. Unlike an earlier review from the same institution, through-the-scope balloon dilators had fewer complications compared to wire-guided dilators, but this difference was not statistically significant ${ }^{86}$ In one meta-analysis consisting of 860 patients undergoing 992 dilations, the risk of postprocedural hemorrhage was $0.1 \%$ and perforation was $0.3 \% .{ }^{83}$ A retrospective chart review and other similar studies have found that there is no correlation between eosinophil count and risk of perforation. ${ }^{87}$ In summary, these single-center studies and systematic reviews confirm the overall safety of dilation in EoE.

\section{Management of refractory EoE}

Refractory EoE is defined as the persistence of clinical symptoms and histologic inflammation despite conventional therapy following PPI, topical steroids, and specialized diets. Its management is challenging. The initial approach should focus on the reasons for treatment failure. This includes a thorough review of initial diagnostic testing for confirmation of diagnosis and excluding mimicking conditions, followed by performing a comprehensive review of the patient's treatment history along with adherence to therapy. This is particularly true for specialized diets, as clinicians need to ensure that patients were fully adherent to therapy with avoidance of potential triggers for an adequate period of time. At times, accidental exposures or contamination from cooking utensils or cookware such as pots or pans can occur. Eating at restaurants is challenging during food elimination diets for EoE. Consultation with an allergist and nutritionist with expertise in this area is recommended for EoE patients managed with specialized diets. Surgical procedures do not have a role in the management of EoE.

Systemic corticosteroid therapy, particularly oral prednisone, has been proposed as a salvage therapy for inducing remission in topical steroid-refractory EoE. The only randomized clinical trial to compare prednisone to swallowed fluticasone demonstrated similar results for both. ${ }^{88}$ Given its side-effect profile, prednisone is rarely used in the treatment of EoE, especially long term.

Multiple novel monoclonal antibody therapies have been proposed and several are in various stages of clinical trials. Targets of these therapies include various inflammatory mediators including IL-5, IL-11, IL-13, and CRTH2. Some 
of these novel agents have shown promising results, but none of these agents are commercially available for the treatment of EoE. In particular, each of the trials including an IL-13 inhibitor and CRTH2 inhibitor showed significant histologic response, but moderate clinical benefit in patients with refractory EoE.$^{89}$ Both reslizumab and mepolizumab are monoclonal antibodies targeting IL-5, which plays a role in eosinophil activation. Both these agents have demonstrated significant improvement in eosinophilia; however, no significant effects on symptoms were observed when compared to placebo. ${ }^{20,21}$ Targeted immunotherapies show promise with intriguing possibilities and ultimately may play an important role in the treatment of EoE. However, to date, none of these therapies has established a clear role in clinical practice or become widely commercially available. A large multicenter trial using an IL-13 inhibitor is underway (NCT02098473).

\section{Conclusion}

Various management options are available for EoE. The treatment involves a multidisciplinary approach and consists of specialized diets with either aminoacid-based elemental diets, allergy test-targeted elimination diet, or empiric food elimination. Topical corticosteroids remain first-line treatment, and emerging data suggest maintenance therapy may decrease progression of disease from inflammatory to fibrostenosis. When strictures develop, dilation should be performed as adjunctive therapy with a goal of improving esophageal diameter and reducing frequency of dysphagia and food impactions. Defining treatment end points remains a challenge. Currently, a multifaceted approach to predict end points include histology, clinical symptoms, and endoscopic features. Management of refractory EoE poses a challenge to clinicians and adherence to therapy should be emphasized. Newer drugs on the horizon may be a viable solution for such patients.

\section{Disclosure}

The authors report no conflicts of interest in this work.

\section{References}

1. Dellon ES, Gonsalves N, Hirano I, et al. ACG clinical guideline: evidenced based approach to the diagnosis and management of esophageal eosinophilia and eosinophilic esophagitis (EoE). Am J Gastroenterol. 2013;108:679-692; quiz 693.

2. Lipka S, Keshishian J, Boyce HW, Estores D, Richter JE. The natural history of steroid-naive eosinophilic esophagitis in adults treated with endoscopic dilation and proton pump inhibitor therapy over a mean duration of nearly 14 years. Gastrointest Endosc. 2014;80:592-598.

3. Keshishian J, Vrcel V, Boyce HW, Estores D, Serrano J, Richter JE. Eosinophilic esophagitis: a paradigm shift for pathology. J Clin Gastroenterol. 2014;48:607-612.
4. Schoepfer AM, Safroneeva E, Bussmann C, et al. Delay in diagnosis of eosinophilic esophagitis increases risk for stricture formation in a time-dependent manner. Gastroenterology. 2013;145(6):1230-1236. e1-2.

5. Kuchen T, Straumann A, Safroneeva E, et al. Swallowed topical corticosteroids reduce the risk for long-lasting bolus impactions in eosinophilic esophagitis. Allergy. 2014;69:1248-1254.

6. Singla MB, Moawad FJ. An overview of the diagnosis and management of eosinophilic esophagitis. Clin Transl Gastroenterol. 2016;7:e155.

7. Straumann A. Treatment of eosinophilic esophagitis: diet, drugs, or dilation? Gastroenterology. 2012;142:1409-1411.

8. Pentiuk S, Putnam PE, Collins MH, Rothenberg ME. Dissociation between symptoms and histological severity in pediatric eosinophilic esophagitis. J Pediatr Gastroenterol Nutr. 2009;48:152-160.

9. Safroneeva E, Straumann A, Coslovsky M, et al. Symptoms have modest accuracy in detecting endoscopic and histologic remission in adults with eosinophilic esophagitis. Gastroenterology. 2016;150:581-590. e4.

10. Collins MH, Martin LJ, Alexander ES, et al. Newly developed and validated eosinophilic esophagitis histology scoring system and evidence that it outperforms peak eosinophil count for disease diagnosis and monitoring. Dis Esophagus. Epub 2016 Feb 9.

11. Hirano I, Moy N, Heckman MG, Thomas CS, Gonsalves N, Achem SR. Endoscopic assessment of the oesophageal features of eosinophilic oesophagitis: validation of a novel classification and grading system. Gut. 2013;62:489-495.

12. Yantiss RK. Eosinophils in the GI tract: how many is too many and what do they mean? Mod Pathol. 2015;28(Suppl 1):S7-S21.

13. Konikoff MR, Noel RJ, Blanchard C, et al. A randomized, doubleblind, placebo-controlled trial of fluticasone propionate for pediatric eosinophilic esophagitis. Gastroenterology. 2006;131:1381-1391.

14. Peterson KA, Thomas KL, Hilden K, Emerson LL, Wills JC, Fang JC. Comparison of esomeprazole to aerosolized, swallowed fluticasone for eosinophilic esophagitis. Dig Dis Sci. 2010;55:1313-1319.

15. Gupta SK, Vitanza JM, Collins MH. Efficacy and safety of oral budesonide suspension in pediatric patients with eosinophilic esophagitis. Clin Gastroenterol Hepatol. 2015;13:66-76. e3.

16. Gonsalves N, Yang GY, Doerfler B, Ritz S, Ditto AM, Hirano I. Elimination diet effectively treats eosinophilic esophagitis in adults: food reintroduction identifies causative factors. Gastroenterology. 2012;142:1451-1459. e1; quiz e14-5.

17. Alexander JA, Jung KW, Arora AS, et al. Swallowed fluticasone improves histologic but not symptomatic response of adults with eosinophilic esophagitis. Clin Gastroenterol Hepatol. 2012;10: 742-749. e1.

18. Dellon ES, Aderoju A, Woosley JT, Sandler RS, Shaheen NJ. Variability in diagnostic criteria for eosinophilic esophagitis: a systematic review. Am J Gastroenterol. 2007;102:2300-2313.

19. Dellon ES, Speck O, Woodward K, et al. Distribution and variability of esophageal eosinophilia in patients undergoing upper endoscopy. Mod Pathol. 2015;28:383-390.

20. Spergel JM, Rothenberg ME, Collins MH, et al. Reslizumab in children and adolescents with eosinophilic esophagitis: results of a doubleblind, randomized, placebo-controlled trial. J Allergy Clin Immunol. 2012;129:456-463, 463. e1-3.

21. Assa'ad AH, Gupta SK, Collins MH, et al. An antibody against IL-5 reduces numbers of esophageal intraepithelial eosinophils in children with eosinophilic esophagitis. Gastroenterology. 2011;141:1593-1604.

22. Rothenberg ME, Wen T, Greenberg A, et al. Intravenous anti-IL-13 mAb QAX576 for the treatment of eosinophilic esophagitis. J Allergy Clin Immunol. 2015;135:500-507.

23. Schoepfer AM, Straumann A, Panczak R, et al. Development and validation of a symptom-based activity index for adults with eosinophilic esophagitis. Gastroenterology. 2014;147:1255-1266. e21.

24. Dellon ES, Irani AM, Hill MR, Hirano I. Development and field testing of a novel patient-reported outcome measure of dysphagia in patients with eosinophilic esophagitis. Aliment Pharmacol Ther. 2013;38:634-642. 
25. Grudell AB, Alexander JA, Enders FB, et al. Validation of the Mayo Dysphagia Questionnaire. Dis Esophagus. 2007;20:202-205.

26. Moawad FJ, Veerappan GR, Dias JA, Baker TP, Maydonovitch CL, Wong RK. Randomized controlled trial comparing aerosolized swallowed fluticasone to esomeprazole for esophageal eosinophilia. Am J Gastroenterol. 2013;108:366-372.

27. Straumann A, Conus S, Degen L, et al. Budesonide is effective in adolescent and adult patients with active eosinophilic esophagitis. Gastroenterology. 2010;139:1526-1537, 1537. e1.

28. Straumann A, Blanchard C, Radonjic-Hoesli S, et al. A new eosinophilic esophagitis (EoE)-like disease without tissue eosinophilia found in EoE families. Allergy. 2016;71(6):889-900.

29. Singla MB, Chehade M, Brizuela D, et al. Early comparison of inflammatory vs. fibrostenotic phenotype in eosinophilic esophagitis in a multicenter longitudinal study. Clin Transl Gastroenterol. 2015;6:e132.

30. van Rhijn BD, Verheij J, van den Bergh Weerman MA, et al. Histological response to fluticasone propionate in patients with eosinophilic esophagitis is associated with improved functional esophageal mucosal integrity. Am J Gastroenterol. 2015;110(9):1289-1297.

31. Dranove JE, Horn DS, Davis MA, Kernek KM, Gupta SK. Predictors of response to proton pump inhibitor therapy among children with significant esophageal eosinophilia. J Pediatr. 2009;154:96-100.

32. Francis DL, Foxx-Orenstein A, Arora AS, et al. Results of ambulatory $\mathrm{pH}$ monitoring do not reliably predict response to therapy in patients with eosinophilic oesophagitis. Aliment Pharmacol Ther. 2012;35:300-307.

33. Lucendo AJ, Arias A, Molina-Infante J. Efficacy of proton pump inhibitor drugs for inducing clinical and histologic remission in patients with symptomatic esophageal eosinophilia: a systematic review and metaanalysis. Clin Gastroenterol Hepatol. 2016;14:13-22.e1.

34. Molina-Infante J, Bredenoord AJ, Cheng E, et al. Proton pump inhibitorresponsive oesophageal eosinophilia: an entity challenging current diagnostic criteria for eosinophilic oesophagitis. Gut. 2016;65:524-531.

35. Dellon ES, Speck O, Woodward K, et al. Clinical and endoscopic characteristics do not reliably differentiate PPI-responsive esophageal eosinophilia and eosinophilic esophagitis in patients undergoing upper endoscopy: a prospective cohort study. Am J Gastroenterol. 2013;108:1854-1860.

36. Moawad FJ, Wells JM, Johnson RL, Reinhardt BJ, Maydonovitch CL, Baker TP. Comparison of eotaxin-3 biomarker in patients with eosinophilic oesophagitis, proton pump inhibitor-responsive oesophageal eosinophilia and gastro-oesophageal reflux disease. Aliment Pharmacol Ther. 2015;42:231-238.

37. Wen T, Dellon ES, Moawad FJ, Furuta GT, Aceves SS, Rothenberg ME. Transcriptome analysis of proton pump inhibitor-responsive esophageal eosinophilia reveals proton pump inhibitor-reversible allergic inflammation. J Allergy Clin Immunol. 2015;135:187-197.

38. Dellon ES, Speck O, Woodward K, et al. Markers of eosinophilic inflammation for diagnosis of eosinophilic esophagitis and proton pump inhibitor-responsive esophageal eosinophilia: a prospective study. Clin Gastroenterol Hepatol. 2014;12:2015-2022.

39. Moawad FJ, Schoepfer AM, Safroneeva E, et al. Eosinophilic oesophagitis and proton pump inhibitor-responsive oesophageal eosinophilia have similar clinical, endoscopic and histological findings. Aliment Pharmacol Ther. 2014;39:603-608.

40. Cheng E, Souza RF, Spechler SJ. Eosinophilic esophagitis: interactions with gastroesophageal reflux disease. Gastroenterol Clin North Am. 2014;43:243-256.

41. van Rhijn BD, Weijenborg PW, Verheij J, et al. Proton pump inhibitors partially restore mucosal integrity in patients with proton pump inhibitor-responsive esophageal eosinophilia but not eosinophilic esophagitis. Clin Gastroenterol Hepatol. 2014;12:1815-1823. e2.

42. Zhang X, Cheng E, Huo X, et al. Omeprazole blocks STAT6 binding to the eotaxin-3 promoter in eosinophilic esophagitis cells. PLoS One. 2012;7:e50037.

43. Cheng E, Zhang X, Huo X, et al. Omeprazole blocks eotaxin-3 expression by oesophageal squamous cells from patients with eosinophilic oesophagitis and GORD. Gut. 2013;62:824-32.
44. Dohil R, Newbury RO, Aceves S. Transient PPI responsive esophageal eosinophilia may be a clinical sub-phenotype of pediatric eosinophilic esophagitis. Dig Dis Sci. 2012;57:1413-1419.

45. Molina-Infante J, Rodriguez-Sanchez J, Martinek J, et al. Long-term loss of response in proton pump inhibitor-responsive esophageal eosinophilia is uncommon and influenced by CYP2C19 genotype and rhinoconjunctivitis. Am J Gastroenterol. 2015;110:1567-1575.

46. Dellon ES, Kim HP, Sperry SL, Rybnicek DA, Woosley JT, Shaheen NJ. A phenotypic analysis shows that eosinophilic esophagitis is a progressive fibrostenotic disease. Gastrointest Endosc. 2014;79: $577-585 . e 4$

47. Lipka S, Kumar A, Richter JE. Impact of diagnostic delay and other risk factors on eosinophilic esophagitis phenotype and esophageal diameter. J Clin Gastroenterol. 2016;50:134-140.

48. Lipka S, Boyce HW, Kumar A, Richter JE. The changing faces of eosinophilic esophagitis: the impact of consensus guidelines at the University of South Florida. Dig Dis Sci. 2015;60:1572-1578.

49. Eluri S, Runge TM, Cotton CC, et al. The extremely narrow-caliber esophagus is a treatment-resistant subphenotype of eosinophilic esophagitis. Gastrointest Endosc. 2016;83:1142-1148.

50. Greenhawt M, Aceves SS, Spergel JM, et al. The management of eosinophilic esophagitis. JAllergy Clin Immunol Pract. 2013;1:332-340; quiz 341-342.

51. Noel RJ, Putnam PE, Rothenberg ME. Eosinophilic esophagitis. NEngl J Med. 2004;351:940-941.

52. Arora AS, Perrault J, Smyrk TC. Topical corticosteroid treatment of dysphagia due to eosinophilic esophagitis in adults. Mayo Clin Proc. 2003;78:830-835.

53. Remedios M, Campbell C, Jones DM, Kerlin P. Eosinophilic esophagitis in adults: clinical, endoscopic, histologic findings, and response to treatment with fluticasone propionate. Gastrointest Endosc. 2006;63:3-12.

54. Butz BK, Wen T, Gleich GJ, et al. Efficacy, dose reduction, and resistance to high-dose fluticasone in patients with eosinophilic esophagitis. Gastroenterology. 2014;147:324-333. e5.

55. Miehlke S, Hruz P, Vieth M, et al. A randomised, double-blind trial comparing budesonide formulations and dosages for short-term treatment of eosinophilic oesophagitis. Gut. 2016;65:390-399.

56. Dellon ES, Sheikh A, Speck O, et al. Viscous topical is more effective than nebulized steroid therapy for patients with eosinophilic esophagitis. Gastroenterology. 2012;143:321-324. e1.

57. Dohil R, Newbury R, Fox L, Bastian J, Aceves S. Oral viscous budesonide is effective in children with eosinophilic esophagitis in a randomized, placebo-controlled trial. Gastroenterology. 2010;139:418-429.

58. Aceves SS, Bastian JF, Newbury RO, Dohil R. Oral viscous budesonide: a potential new therapy for eosinophilic esophagitis in children. $\mathrm{Am} \mathrm{J}$ Gastroenterol. 2007;102:2271-2279; quiz 2280.

59. Albert D, Heifert TA, Min SB, et al. Comparisons of fluticasone to budesonide in the treatment of eosinophilic esophagitis. Dig Dis Sci. 2016;61(7):1996-2001.

60. Philla KQ, Min SB, Hefner JN, et al. Swallowed glucocorticoid therapy for eosinophilic esophagitis in children does not suppress adrenal function. J Pediatr Endocrinol Metab. 2015;28:1101-1106.

61. Harel S, Hursh BE, Chan ES, Avinashi V, Panagiotopoulos C. Adrenal suppression in children treated with oral viscous budesonide for eosinophilic esophagitis. J Pediatr Gastroenterol Nutr. 2015;61:190-193.

62. Boldorini R, Mercalli F, Oderda G. Eosinophilic oesophagitis in children: responders and non-responders to swallowed fluticasone. J Clin Pathol. 2013;66:399-402.

63. Wolf WA, Cotton CC, Green DJ, et al. Predictors of response to steroid therapy for eosinophilic esophagitis and treatment of steroid-refractory patients. Clin Gastroenterol Hepatol. 2015;13:452-458.

64. Straumann A, Spichtin HP, Grize L, Bucher KA, Beglinger C, Simon HU. Natural history of primary eosinophilic esophagitis: a follow-up of 30 adult patients for up to 11.5 years. Gastroenterology. 2003;125: $1660-1669$

65. Aceves SS, Newbury RO, Dohil R, Bastian JF, Broide DH. Esophageal remodeling in pediatric eosinophilic esophagitis. J Allergy Clin Immunol. 2007;119:206-212. 
66. Straumann A, Conus S, Degen L, et al. Long-term budesonide maintenance treatment is partially effective for patients with eosinophilic esophagitis. Clin Gastroenterol Hepatol. 2011;9:400-409. e1.

67. Aceves SS, Newbury RO, Chen D, et al. Resolution of remodeling in eosinophilic esophagitis correlates with epithelial response to topical corticosteroids. Allergy. 2010;65:109-116.

68. Arias A, Gonzalez-Cervera J, Tenias JM, Lucendo AJ. Efficacy of dietary interventions for inducing histologic remission in patients with eosinophilic esophagitis: a systematic review and meta-analysis. Gastroenterology. 2014;146:1639-1648.

69. Markowitz JE, Spergel JM, Ruchelli E, Liacouras CA. Elemental diet is an effective treatment for eosinophilic esophagitis in children and adolescents. Am J Gastroenterol. 2003;98:777-782.

70. Peterson KA, Byrne KR, Vinson LA, et al. Elemental diet induces histologic response in adult eosinophilic esophagitis. Am J Gastroenterol. 2013;108:759-766.

71. Doerfler B, Bryce P, Hirano I, Gonsalves N. Practical approach to implementing dietary therapy in adults with eosinophilic esophagitis: the Chicago experience. Dis Esophagus. 2015;28:42-58.

72. Arias A, Lucendo AJ. Dietary therapies for eosinophilic esophagitis. Expert Rev Clin Immunol. 2014;10:133-142.

73. Spergel JM, Andrews T, Brown-Whitehorn TF, Beausoleil JL, Liacouras CA.. Treatment of eosinophilic esophagitis with specific food elimination diet directed by a combination of skin prick and patch tests. Ann Allergy Asthma Immunol. 2005;95:336-343.

74. Wolf WA, Jerath MR, Sperry SL, et al. Dietary elimination therapy is an effective option for adults with eosinophilic esophagitis. Clin Gastroenterol Hepatol. 2014;12:1272-9.

75. Spergel JM, Brown-Whitehorn TF, Cianferoni A, Shaheen NJ, Dellon ES. Identification of causative foods in children with eosinophilic esophagitis treated with an elimination diet. J Allergy Clin Immunol. 2012;130:461-467.e5.

76. Henderson CJ, Abonia JP, King EC, et al. Comparative dietary therapy effectiveness in remission of pediatric eosinophilic esophagitis. J Allergy Clin Immunol. 2012;129:1570-1578.

77. Lucendo AJ, Arias A, Gonzalez-Cervera J, et al. Empiric 6-food elimination diet induced and maintained prolonged remission in patients with adult eosinophilic esophagitis: a prospective study on the food cause of the disease. J Allergy Clin Immunol. 2013;131:797-804.
78. Molina-Infante J, Arias A, Barrio J, Rodríguez-Sánchez J, SanchezCazalilla M, Lucendo AJ. Four-food group elimination diet for adult eosinophilic esophagitis: a prospective multicenter study. JAllergy Clin Immunol. 2014;134:1093-1099. e1.

79. Lucendo AJ, De Rezende L. Endoscopic dilation in eosinophilic esophagitis: a treatment strategy associated with a high risk of perforation. Endoscopy. 2007;39:376; author reply 377.

80. Cohen MS, Kaufman AB, Palazzo JP, et al. An audit of endoscopic complications in adult eosinophilic esophagitis. Clin Gastroenterol Hepatol. 2007;5:1149-1153.

81. Schoepfer AM, Gonsalves N, Bussmann C, et al. Esophageal dilation in eosinophilic esophagitis: effectiveness, safety, and impact on the underlying inflammation. Am J Gastroenterol. 2010;105: 1062-1070.

82. Bohm ME, Richter JE. Review article: oesophageal dilation in adults with eosinophilic oesophagitis. Aliment Pharmacol Ther. 2011;33:748-757.

83. Moawad FJ, Cheatham JG, DeZee KJ. Meta-analysis: the safety and efficacy of dilation in eosinophilic oesophagitis. Aliment Pharmacol Ther. 2013;38:713-720.

84. Dellon ES, Gibbs WB, Rubinas TC, et al. Esophageal dilation in eosinophilic esophagitis: safety and predictors of clinical response and complications. Gastrointest Endosc. 2010;71:706-712.

85. Jung KW, Gundersen N, Kopacova J, et al. Occurrence of and risk factors for complications after endoscopic dilation in eosinophilic esophagitis. Gastrointest Endosc. 2011;73:15-21.

86. Runge TM, Eluri S, Cotton CC, et al. Outcomes of esophageal dilation in eosinophilic esophagitis: safety, efficacy, and persistence of the fibrostenotic phenotype. Am J Gastroenterol. 2016;111:206-213.

87. Ukleja A, Shiroky J, Agarwal A, Allende D. Esophageal dilations in eosinophilic esophagitis: a single center experience. World J Gastroenterol. 2014;20:9549-9555.

88. Schaefer ET, Fitzgerald JF, Molleston JP, et al. Comparison of oral prednisone and topical fluticasone in the treatment of eosinophilic esophagitis: a randomized trial in children. Clin Gastroenterol Hepatol. 2008;6:165-73.

89. Straumann A, Hoesli S, Bussmann C, et al. Anti-eosinophil activity and clinical efficacy of the CRTH2 antagonist OC000459 in eosinophilic esophagitis. Allergy. 2013;68:375-385.
Clinical and Experimental Gastroenterology

\section{Publish your work in this journal}

Clinical and Experimental Gastroenterology is an international, peerreviewed, open access, online journal publishing original research, reports, editorials, reviews and commentaries on all aspects of gastroenterology in the clinic and laboratory. This journal is included on PubMed. The manuscript management system is completely online

\section{Dovepress}

and includes a very quick and fair peer-review system, which is all easy to use. Visit http://www.dovepress.com/testimonials.php to read real quotes from published authors. 